\section{Copeptin for risk stratification in acute illness: beyond cardiological problems}

\author{
Roberto Cemin, ${ }^{1}$ Giuseppe Lippi, ${ }^{2}$ \\ Luca Donazzan, ${ }^{1}$ Massimiliano Manfrin, \\ Massimo Daves ${ }^{3}$ \\ 'Department of Cardiology, San Maurizio \\ Regional Hospital of Bolzano, Bolzano; \\ ${ }^{2}$ Laboratory of Clinical Chemistry and \\ Hematology, Parma University Hospital, \\ Parma; ${ }^{3}$ Clinical Biochemistry Laboratory, \\ San Maurizio Regional Hospital of \\ Bolzano, Bolzano, Italy
}

\section{Abstract}

Copeptin (Cop) has been recently proposed as a reliable marker for the diagnosis of acute coronary syndrome, altough its concentration was found to increase in a variety of other severe clinical conditions. The aim of the present study was to assess the utility of Cop to identify high-risk patients in the emergency room (ER). Eighty-five patients admitted to ER of the San Maurizio Regional Hospital of Bolzano between February to March 2010 with epigastric or chest pain and/or discomfort were included in the study. Blood was drawn at admission and sampled for Cop in standard laboratory tests. Cop levels were significantly higher in patients who died at the hospital or shortly afterwards as compared with survivors (median 61 vs $40.6 \mathrm{pmol} / \mathrm{L} ; \mathrm{P}=0.014$ ). Cop levels were also higher in patients with severe health problems $(62.9$ vs $28.3 \mathrm{pmol} / \mathrm{L}$; $\mathrm{P}<0.0001)$. The area under the receiver-operating characteristic curve of Cop was 0.70 for in-hospital death [95\% confidence interval (CI) $0.53-0.86$ ], 0.74 for acute and subacute death (95\% CI $0.61-0.87)$ and 0.90 for prediction of severe acute illness (95\% CI 0.84-0.97). Accordingly, a Cop level $>33.1 \mathrm{pmol} / \mathrm{L}$ correctly identified in-hospital death with $71 \%$ sensitivity and $74 \%$ specificity. A Cop level $>13.6$ $\mathrm{pmol} / \mathrm{L}$ was instead associated with $89 \%$ sensitivity and $80 \%$ specificity for identifying patients with acute and severe conditions. The results of our analysis would suggest that the use of Cop may be a valuable aid in the ER for identifying patients with life-threatening conditions.

\section{Introduction}

Copeptin (Cop) has been recently proposed as a reliable marker to be used in combination with cardiac troponins I or T (i.e. cTnI or cTnT, respectively) as an aid in early diagnosis of acute coronary syndrome (ACS). In fact the improved diagnostic accuracy of this association has been reliably demonstrated in several studies. $^{1-3}$

Copeptin (i.e. the C-terminal part of the vasopressin prohormone) is a high non-specific marker for myocardial damage, whilst it simpy behaves as an index of hyperactivation of the hypothalamo-pituitary-adrenal axis, thus correlating with individual stress levels. Copeptin is secreted stoichiometrically with arginine-vasopressine and its release is regulated by hyperosmolality, hypovolemia, hypotension, hipotalamic osmoreceptors and angiotensin II. ${ }^{4}$ The concentration of Cop has been found to be increased in several high risk pathologies such as heart failure ${ }^{5-7}$ septic shock ${ }^{8,9}$ stroke, ${ }^{10}$ pneumonia ${ }^{11}$ or after resuscitation from a cardiac arrest, ${ }^{12}$ in which conditions it appear to be a reliable prognostic marker. Copeptin was found to accurately mirror disease severity and to discriminate patients with unfavorable outcomes from those with favorable outcome. ${ }^{13}$ Therefore, Cop may be used as a good prognostic index to predict outcome in acute illness. ${ }^{13}$

The purpose of the present study was to test the utility of Cop assessment in the emergency room (ER) for identifying high-risk patients.

\section{Materials and Methods}

\section{Study population}

Eighty-five patients admitted to the ER of San Maurizio Regional Hospital of Bolzano between February and March 2010 with epigastric or chest pain or discomfort were included in this study. No exclusion criteria were used in order to enrol an unbiased population that would reflect the typical setting of an ER. Blood was drawn at admission by experienced nurses. At the same time of sampling for Cop, blood was sent for standard laboratory test and for measurement of both cTnI and myoglobin, as for usual internal protocol.

Cop samples were stored at $-40^{\circ} \mathrm{C}$ and analyzed within 45 days in a single session in order to avoid calibration influences, so that Cop concentration was blinded at the time of patients management.

The research was conducted in compliance with the Helsinki declaration. The patients gave their consent to the analysis of Cop and were informed that the clinical management would have been blinded from Cop concentration. Since Cop analysis did not affect the clinical decision making and was performed on blood after all request testing had been performed, approval from ethical committee was deemed unnecessary.

Patients, included those directly discharged
Correspondence: Roberto Cemin, Department of Cardiology, San Maurizio Regional Hospital of Bolzano, via L. Boehler 5, 39100 Bolzano, Italy. Tel. +39.0471.909950 - Fax: +39.0471.909977.

E-mail: roberto.cemin@asbz.it

Key words: copeptin, risk stratification, emergency room.

Contributions: RC conceived the study, analyzed the data, wrote the manuscript, searched the references; GL reviewed the manuscript; LD and MM collected the data; MD conceived the study, performed the laboratory analisis, reviewed the manuscript.

Conflict of interests: the authors declare no potential conflict of interests.

Received for publication: 15 February 2013.

Revision received: 2 July 2013.

Accepted for publication: 3 July 2013.

This work is licensed under a Creative Commons Attribution 3.0 License (by-nc 3.0).

(C) Copyright R. Cemin et al., 2013

Licensee PAGEPress, Italy

Emergency Care Journal 2013; 9:e19

doi:10.4081/ecj.2013.e19

from the ER, were further classified as having a severe health problem (Group 1) or not (Group 2), according to the type of diagnosis and treatment. Acute illness was considered severe when shortly conducting to death, requiring a surgical act, intensive care or a prolonged hospital stay (i.e. $>4$ days). Patients were considered at low risk if directly discharged from the ER or quickly ( $<4$ days) from the hospital wards or being suffering from a benign pathology accordingly to two independent observers.

\section{Laboratory methods}

The concentration of cTnI was measured with a double side (sandwich) chemiluminescent immunoenzymatic assay (Access AccuTnI; Beckman Coulter Inc., Brea, CA, USA) on the analyser Access 2 (Beckman Coulter Inc.) according to manufacturer's instructions. Although the detection limit of this assay is $0.01 \mathrm{ng} / \mathrm{mL}$ and the $99^{\text {th }}$ percentile in an apparently healthy reference population is reported to be $0.04 \mathrm{ng} / \mathrm{mL}$, the analysis for this study was planned at a decision limit of $0.06 \mathrm{ng} / \mathrm{mL}$, which corresponds at the $10 \%$ total coefficient of variation (CV) of this immunoassay, as currently recommended. ${ }^{14}$

For cTnI and Cop measurements venous blood samples were collected into siliconised vacuum tubes containing lithium heparin (Terumo Europe N.V., Leuven, Belgium) and centrifuged at room temperature at $1500 \mathrm{xg}$ for 
10 min. Copeptin was measured with a sandwich fluoroimmunoassay assay (B.R.A.H.M.S. Copeptin KRYPTOR; B.R.A.H.M.S GmbH, Hennigsdorf, Germany). Cop samples were obtained from discharged plasma samples used for cTnI determination, stored at $-40^{\circ} \mathrm{C}$ and analyzed within 45 days in a single analytical session. All the measurements were performed on the analyzer KRYPTOR Compact (B.R.A.H.M.S GmbH). The upper reference limit, reported by manufactures is $19.1 \mathrm{pmol} / \mathrm{L}$ in males and $12.9 \mathrm{pmol} / \mathrm{L}$ in females, respectively.

Copeptin levels below the detection limit were labeled as low from the automatic response and were thereby computed as 4.8 $\mathrm{pmol} / \mathrm{L}$ in statistical analysis, which corresponds to the detection limit reported by manufacture.

\section{Statistical analysis}

Data were analyzed using Microsoft Excel ${ }^{\circledR}$ version and the statistical program, Analyse-it for Microsoft Excel ${ }^{\circledR}$. Mean, median, standard deviation and interquartile range (IQR) were calculated for continuous variables. After testing the correlation between Cop and death or severe health problems by Spearman rank correlation test, agreement between the parameters showing significant correlation was analyzed using Passing and Bablok regression method. The Cusum test for linearity was used to assess the kind of relationship between them. $P$ values $<0.05$ were considered statistically significant.

Variables were tested for normal distribution with the Shapiro Wilk W test. Significance of differences and frequency distribution of values between the groups were assessed by the Mann-Whitney test for continuous variables. The receiver operating characteristic (ROC) curves for prediction of death and severe health problems were calculated.

A further analysis was conducted by splitting the entire patient group into 4 quartiles, based on Cop levels and checking the different incidence of the variables mortality, mortality within 4 months, as well as severe health problem between the quartiles. Repeated analysis of variance (ANOVA) was performed to test differences between the four groups (quartiles) for continuous variables and the Chi-square test was used for categorical variables. Results were finally expressed as median and IQR.

\section{Results}

A total number of 85 patients was included in the study. Median age was 75.9 (IQR 53.0$83.7)$ years; $46(54.1 \%)$ of the patients were men and 39 (45.9\%) women. Baseline characteristics of the study population are reported in
Table 1. Copeptin resulted below the normal reference level (i.e. $<4.8 \mathrm{pmol} / \mathrm{L})$ in $10(11.7 \%)$ patients. Median cop level for whole the entire patient population was $13.6 \mathrm{pmol} / \mathrm{L}$ (IQR 8.253.8). Twenty five (29.4\%) out of the 85 patients, were directly discharged from the ER. The remainders $(60 ; 70.6 \%)$ either died in the ER (one patient) or were admitted to the clinical wards and conventionally treated. Median hospital stay was 3 days (IQR 0-8.2).

During the hospital stay, 6 other patients died, so that the overall number of in-hospital death was 7 (i.e. 8\%). Patients who died had a mean copeptin level of $58.3 \mathrm{pmol} / \mathrm{L}$ compared to $41.6 \mathrm{pmol} / \mathrm{L}$ in survivors (not significant). Three other patients died within 4 months after hospital discharge, due to progression of the initial pathology (i.e. 2 end stage heart failure and 1 end stage renal failure), which caused the initial ER visit.

Copeptin levels in the whole population of deceased patients (acute and sub acute; globally 10 patients) was $61 \mathrm{pmol} / \mathrm{L}$, being statistically significant when compared to long surviving patients $(40.6 \mathrm{pmol} / \mathrm{L} ; \mathrm{P}=0.014)$.

Thirty six of the ER patients were considered as critically ill (42.3\%), while 49 were not (57.6\%). Cop level in the former group (i.e. Group 1) was 62.9 vs $28.3 \mathrm{pmol} / \mathrm{L}$ in the noncritical group (Group 2; $\mathrm{P}<0.0001$ ). Type I myocardial infarction ${ }^{15}$ was diagnosed in one patient only. A complete list of the main diagnoses at discharge is shown in Table 2.

Copeptin did not show a significant correlation with in-hospital death, while it was significantly associated to death within 4 months $(\mathrm{P}=0.0131 ; 95 \mathrm{CI} 0.06-0.46)$ and severe health problems $(\mathrm{P}<0.0001$; CI 0.57-0.79).

Copeptin concentration exhibited a direct

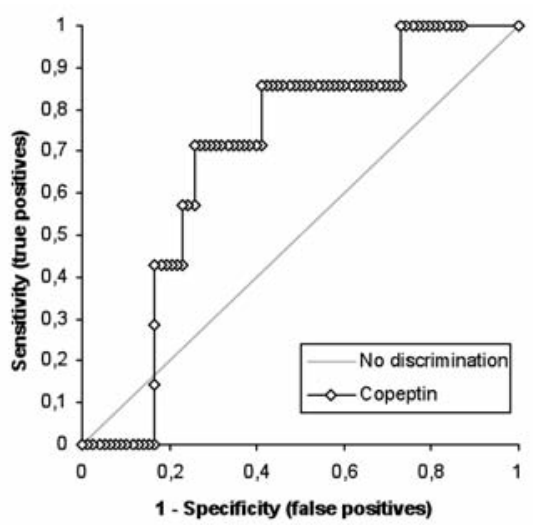

Figure 1. Receiver operating characteristic curve analysis for prediction of death by copeptin on admission to the emergency room. The area under the curve is 0.70 (95\% confidence interval: $0.53-0.86$; $\mathbf{P}=0.0091)$. non-linear relationship with age $(\mathrm{P}<0.01)$, myoglobin $(\mathrm{P}<0.05)$, cTnI $(\mathrm{P}<0.01)$, plasma glucose at ER admission $(\mathrm{P}<0.01)$ and plasma creatinine $(\mathrm{P}<0.01)$, while displaying an inverse non-linear relationship with glomerular filtration rate (GFR), calculated according to the standard modification of diet in renal disease (MDRD) equation $(\mathrm{P}<0.01)$.

As regards the diagnostic accuracy of Cop, the composite score for sensitivity and specificity [i.e. area under the curve (AUC)], determined by ROC curve analysis for prediction of death, death in the following 4 months or severe acute illness are shown in Figures 1,2 and 3 respectively.

Interestingly, ROC curve analysis for prediction of death by admission value of Cop displayed an AUC of 0.70 (95\% CI 0.53-0.86; $\mathrm{P}=0.009$ ), thereby suggesting a statistically significant model, which may be useful in the ER. At the cut-off value of $33.1 \mathrm{pmol} / \mathrm{L}$, sensitivity and specificity were 71 and $74 \%$, respectively. ROC curve analysis for prediction of death in the following 4 months, due to acute problems or progression of end stage pathologies,

Table 1. Baseline characteristics of the study population (85 patients).

Study population $(n=85)$

\begin{tabular}{lc} 
Age (years) & $75.9(53.0-83.7)$ \\
Men/women & $46(54.1 \%) / 39(45.9 \%)$ \\
\hline Cop $(\mathrm{pmol} / \mathrm{L})$ & $13.6(8.25-53.75)$ \\
Dead/hospital survivors & $7(8.2 \%) / 78(91.8 \%)$ \\
\hline cTnI $(\mathrm{ng} / \mathrm{mL})$ & $0.00(0.00-0.02)$ \\
Glycaemia $(\mathrm{mg} / \mathrm{dL})$ & $119(101.75-142.25)$ \\
\hline Creatinine $(\mathrm{mg} / \mathrm{dL})$ & $1.0(0.8-1.22)$ \\
GFR $\left(\mathrm{mL} / \mathrm{min} / 1.73 \mathrm{~m}^{2}\right)$ & $72.9 \pm 30.6$
\end{tabular}

Cop, copeptine; cTnI, cardiac troponin I; GFR, glomerular filtration rate. Data are shown as median and interquartile range in brackets or mean+standard deviation.

Table 2. Main diagnosis at hospital discharge (85 patients, including deaths).

\begin{tabular}{lc} 
Diagnosis & $\begin{array}{c}\text { Study population } \\
(\mathbf{n}=85)\end{array}$ \\
Heart failure & $6(7.1 \%)$ \\
Ischemia* & $6(7.1 \%)$ \\
\hline Infection & $17(20 \%)$ \\
Neoplasia & $8(9.4 \%)$ \\
\hline Trauma & $10(11.8 \%)$ \\
Muscular chest pain & $22(25.9 \%)$ \\
\hline Arrythmias & $7(8.2 \%)$ \\
Aortic dissection & $1(1.1 \%)$ \\
\hline Others $^{\circ}$ & $8(9.4 \%)$ \\
\hline
\end{tabular}

*Ischemia (1 myocardial infarction, 2 strokes, 1 lung embolism, 1 bowel infarction, 1 limb ischemia); ${ }^{\circ}$ others (1 kidney stone, 1 neuropathy, 1 allergic reaction, 1 burn, 3 major hemorrhages, 1 limb edema). 
Table 3. Patients outcome in relation to copeptin quartiles.

\begin{tabular}{|c|c|c|c|c|c|}
\hline & $\begin{array}{l}\text { Quartile } 1 \\
<8.25 \mathrm{pmol} / \mathrm{L} \\
(\mathrm{n}=21)\end{array}$ & $\begin{array}{c}\text { Quartile } 2 \\
\text { 8.26-13.6 pmol/L } \\
(\mathrm{n}=22)\end{array}$ & $\begin{array}{c}\text { Quartile } 3 \\
\text { 13.61-53.7 pmol/L } \\
\text { (n=21) }\end{array}$ & $\begin{array}{l}\text { Quartile } 4 \\
>53.71 \mathrm{pmol} / \mathrm{L} \\
\quad(\mathrm{n}=21)\end{array}$ & $\mathbf{P}$ \\
\hline Age (years) & $61.4 \pm 19.7$ & $57.7 \pm 19.9$ & $71.8 \pm 19.2$ & $81.8 \pm 6.3$ & $<0.0001$ \\
\hline Male & $8(11.4)$ & $12(11.9)$ & 15 (11.4) & $11(11.4)$ & 0.19 \\
\hline Death & $0(1.7)$ & $1(1.8)$ & $3(1.7)$ & $3(1.7)$ & 0.23 \\
\hline Death within 4 months & $0(2.5)$ & $1(2.6)$ & $4(2.5)$ & $5(2.5)$ & $<0.05$ \\
\hline Severe health problems & $0(8.9)$ & $4(9.3)$ & $14(8.9)$ & $18(8.9)$ & $<0.0001$ \\
\hline Glycaemia (mg/dL) & $114.6 \pm 21.9$ & $117.0 \pm 33.2$ & $139.9 \pm 55.1$ & $168.5 \pm 61.3$ & 0.0006 \\
\hline Creatinine (mg/dL) & $0.81 \pm 0.16$ & $0.89 \pm 0.24$ & $1.33 \pm 0.55$ & $1.8 \pm 1.43$ & 0.0002 \\
\hline GFR (mL/min/1.73m²) & $91.7 \pm 28.1$ & $88.3 \pm 22.8$ & $61.15 \pm 24.2$ & $49.55 \pm 25.6$ & $<0.0001$ \\
\hline
\end{tabular}

GFR, glomerular filtration rate. Data are shown as number and number expected in brackets or mean \pm standard deviation.

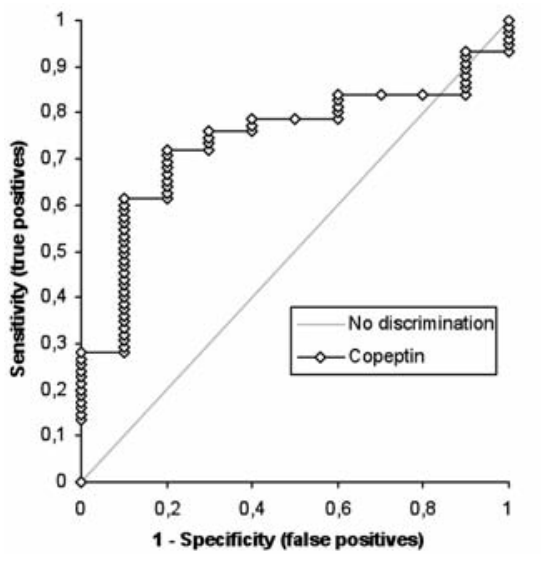

Figure 2. Receiver operating characteristic curve analysis for prediction of death in a 4-month period by copeptin on admission to the emergency room. The area under the curve is $0.74(95 \%$ confidence interval: 0.61-0.87; $P=0.002$ ).

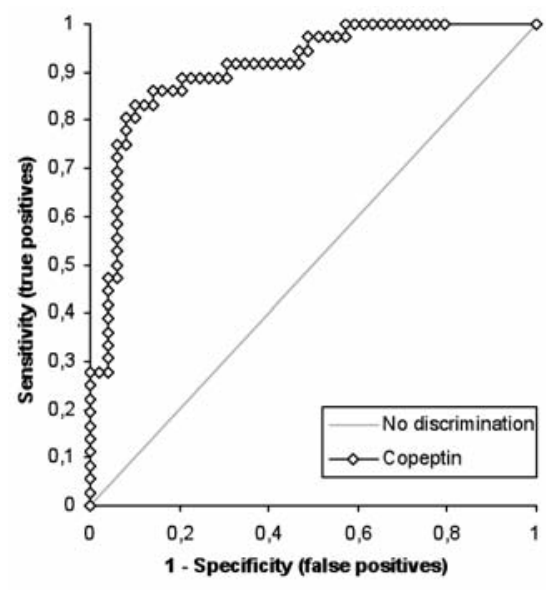

Figure 3. Receiver operating characteristic curve analysis for prediction of severe acute illness by copeptin on admission to the emergency room. The area under the curve is $0.90(95 \%$ confidence interval: $0.84-$ 0.97; $\mathrm{P}<0.0001)$. showed an AUC of 0.74 (95\% CI, 0.61-0.87; $\mathrm{P}=0.002$ ) for by Cop concentration on admission. At cut-off value of $33.1 \mathrm{pmol} / \mathrm{L}$, sensitivity and specificity were 75 and $70 \%$, respectively. The ROC curve analysis for prediction of severe acute illness showed an AUC of 0.90 (95\% CI, 0.84-0.97; $\mathrm{P}<0.0001$ ) for Cop on admission, which is also suggestive of a statistically significant, clinical useful model (e.g. at a cut-off value of $13.6 \mathrm{pmol} / \mathrm{L}$, the sensitivity and specificity were 89 and $80 \%$, respectively).

Patients in different quartiles of Cop were compared according to baseline characteristics and outcome. Patients in the highest quartile were older, had a higher value of plasma glucose and creatinine level, and a lower GFR. The rate of death, and death within 4 months was higher in the third and forth quartiles, as was the prevalence of severe health conditions. The results of quartile analysis are shown in Table 3.

\section{Discussion}

Copeptin has been extensively investigated in patients with cardiac injury and impairment of heart function, and has also been recently proposed as a reliable biomarker for the rule out of AMI in combination with cardiac troponins. Considering the fact that Cop is not specific for cardiac problems, the present study was designed to investigate the role of copeptin in the diagnosis of particularly severe health conditions, in patients acutely admitted to the ER. Our results clearly attest that Cop concentration is remarkably increased in patients at high risk of severe outcomes and/or death. A cut off level of $13.6 \mathrm{pmol} / \mathrm{L}$ had a good prognostic value, exhibiting a remarkable AUC of 0.90 for severe problems.

This threshold closely mirrors that suggested for the rule out of ACS in previous investigations. $^{2}$

A higher cutoff (i.e. $33.1 \mathrm{pmol} / \mathrm{L}$ ) showed however a significant prognostic value for pre- dicting death within 4 months from ER admission, with an AUC of 0.74 , combined with sensitivity of 75 and specificity of $70 \%$.

These results prompt us to suggest that Cop may be a valuable biomarker for improving the prognostication of patients in the ER and may thereby be used for stratification of risk, guiding the clinical decision making, optimizing the therapeutic management of the single patients, and ultimately optimizing the use of healthcare resources. This is particularly important in the ER, wherein simple and economical biomarkers are urgently needed to prevent or limit overcrowding. ${ }^{16}$ Still, it is important to mention here that the interpretation of Cop results must always be evaluated in the context of a careful clinical assessment, since its concentration may be increased for in a variety of clinical conditions. The emergency setting seems to be the ideal place where the measurement of Cop might provide information for risk stratification in all different types of acute illnesses, wherein the use of biomarkers may aid the prognostic assessment.

\section{Conclusions}

The results of our study suggest that Cop may be a useful biomarker in the ER, not only for early diagnosis of ACS, but also for identifying those clinical conditions that mostly jeopardize patient's health. Furthermore, Cop has showed a good prognostic power regarding death. Further studies are needed to confirm our observation in a larger sample of patients.

\section{References}

1. Keller T, Tzikas S, Zeller T, et al. Copeptin improves early diagnosis of acute myocardial infarction. J Am Coll Cardiol 2010;55: 2096-106.

2. Reichlin T, Hochholzer W, Stelzig C, et al. 
Incremental value of copeptin for rapid rule out of acute myocardial infarction. $\mathbf{J}$ Am Coll Cardiol 2009;54:60-8.

3. Lippi G, Plebani M, Di Somma S, et al. Considerations for early acute myocardial infarction rule-out for emergency department chest pain patients: the case of copeptin. Clin Chem Lab Med 2012;50:24353.

4. Katan M, Morgenthaler N, Widmer I, et al. Copeptin, a stable peptide derived from the vasopressin precursor, correlates with the individual stress levels. Neuro Endocrinol Lett 2008;29:341-6.

5. Kelly D, Squire IB, Khan SQ, et al. C-terminal provasopressin (copeptin) is associated with left ventricular dysfunction, remodeling and clinical heart failure in survivors of myocardial infarction. J Card Fail 2008;14:739-45.

6. Stoiser B, Mortl D, Hulsmann M, et al. Copeptin, a fragment of the vasopressin precursor, as a novel predictor of heart outcome in heart failure. Eur J Clin Invest 2006;36:771-8.

7. Maisel A, Xue Y, Shah K, et al. Increased 90-day mortality in patients with acute heart failure with elevated copeptin: secondary results from the biomarkers in acute heart failure (BAHC). Circ Heart Fail 2011;4:613-20.

8. Mutlu GM, Factor P. Role of vasopressin in the management of septic shock. Intens Care Med 2004;30:1276-91.

9. Morgenthaler NG, Mueller B, Struck J, et al. Copeptine, a stable peptide of the arginine vasopressin precursor is elevated in hemorrhagic and septic shock. Shock 2007;28:219-26.

10. Katan M, Fluri F, Morgenthaler NG, et al. Copeptin: a novel, independent prognostic marker in patients with ischemic stroke. Ann Neurol 2009;66:799-808.

11. Muller B, Morgenthaler N, Stolz D, et al. Circulating levels of copeptin, a novel biomarker in lower tract infections. Eur J Clin
Invest 2007;37:145-52.

12. Krismer AC, Wenzel V, Stadlbauer KH, et al. Vasopressin during cardiopulmonary resuscitation. Anesthesiology 1992;77:6628.

13. Katana M, Christ-Craina M. The stress hormone copeptin: a new prognostic biomarker in acute illness. Swiss Med Wkly 2010;140:w13101.

14. Morrow DA, Rifai N, Sabatine MS, et al. Evaluation of the AccuTnI cardiac troponin I assay for risk assessment in acute coronary syndromes. Clin Chem 2003;49:13968.

15. Thygesen K, Alpert JS, White HD, et al. Universal definition of myocardial infarction. Circulation 2007;116:2634-53.

16. Lippi G, Targher G, Neri G, et al. Diagnostic significance of haematological testing in patients presenting at the emergency department. Emerg Care J 2012;8:712. 